\title{
Quantum Painlevé Equations: from Continuous to Discrete
}

\author{
Hajime NAGOYA ${ }^{\dagger}$, Basil GRAMMATICOS ${ }^{\ddagger}$ and Alfred RAMANI ${ }^{\S}$ \\ $\dagger$ Graduate School of Mathematical Sciences, The University of Tokyo, Japan \\ E-mail: nagoya@ms.u-tokyo.ac.jp \\ ‡ IMNC, Université Paris VII \& XI, CNRS, UMR 8165, Bât. 104, 91406 Orsay, France \\ E-mail: grammati@paris7.jussieu.fr \\ $\S$ Centre de Physique Théorique, Ecole Polytechnique, CNRS, 91128 Palaiseau, France \\ E-mail: ramani@cpht.polytechnique.fr
}

Received March 05, 2008, in final form May 03, 2008; Published online June 09, 2008

Original article is available at http://www.emis.de/journals/SIGMA/2008/051/

\begin{abstract}
We examine quantum extensions of the continuous Painlevé equations, expressed as systems of first-order differential equations for non-commuting objects. We focus on the Painlevé equations II, IV and V. From their auto-Bäcklund transformations we derive the contiguity relations which we interpret as the quantum analogues of the discrete Painlevé equations.
\end{abstract}

Key words: discrete systems; quantization; Painlevé equations

2000 Mathematics Subject Classification: 34M55; 37K55; 81S99

\section{Introduction}

The word "quantum" used in the title needs some qualifying. Historically, this term was introduced in relation to the discreteness of the spectrum of operators like the Hamiltonian and the angular momentum. However, with the blossoming of quantum mechanics, its use was generalised to the description of non-classical objects, typically non-commuting operators. It is in this sense that we are going to use the term quantum in this paper: namely, for the description of equations where the various components of the dependent variable do not commute among themselves.

In this paper we shall focus on integrable equations involving non-commuting variables. Their interest, in particular, as far as quantum field theories are concerned, is obvious. A large literature exists concerning supersymmetric or just fermionic extensions of integrable evolution equations. It is now clear that the special properties which characterise integrability can be extended to the case where the dependent variable involves fermionic as well as bosonic components.

The quantisation of low dimensional integrable Hamiltonian systems has been the object of extensive investigations by Hietarinta and collaborators $[6,7]$. It was shown, in particular, that the preservation of integrability in a quantum setting often necessitates the introduction of purely quantum (i.e., explicitly $\hbar$ dependent) terms in both the Hamiltonian and the invariants. In some cases, these terms can be absorbed by the proper ordering and the introduction of a non-flat space metric. However, it is not clear whether this suffices in all cases.

The "quantum" extension of (continuous) Painlevé equations has been introduced by one of us $(\mathrm{HN})$ in $[9,10]$. For these paradigmatic integrable systems, integrability is not related to the existence of invariants, but rather to the fact that these nonautonomous equations can be obtained as the compatibility condition of a (an overdetermined) linear system, the Lax pair. 
(Incidentally, Novikov [12] refers to this compatibility condition as a quantisation condition for the spectral curve and thus the deautonomisation process can be considered, in some formal sense, as a first kind of quantisation.)

Starting from "quantum" continuous Painlevé equations we shall derive their contiguity relations which, as already shown in the commuting case, can be interpreted as discrete Painlevé equations $[1,8]$. Quantum and discrete systems possess a common character as far as the phasespace of their dynamics is concerned. While in the former case the surface of an elementary cell is fixed by the relation $\Delta x \Delta p=\hbar$ in the latter case the elementary cell is rigidly fixed by $\Delta x=\Delta p=1$ (in the appropriate units). The main difficulty in quantising discrete systems, integrability notwithstanding, lies in the fact that one must introduce a commutation rule consistent with the evolution [13]. This is a highly nontrivial problem. We have addressed this question in [4] and [16] where we have shown that for the mappings of the QRT [14] family the following commutation rule

$$
x y=q y x+\lambda x+\mu y+\nu
$$

is consistent with the evolution (with the adequate choice of the parameters). Since discrete Painlevé equations are nonautonomous extensions of the QRT mappings we expect rule (1) to be sufficient for the quantisation of the cases we shall consider here.

In what follows, we shall analyse the "quantum" forms of continuous Painlevé equations derived by one of us (HN) in [9] and use their auto-Bäcklund transformations so as to deduce their contiguity relations. Thus working with quantum forms of $\mathrm{P}_{\mathrm{II}}, \mathrm{P}_{\mathrm{IV}}$ and $\mathrm{P}_{\mathrm{V}}$ we will derive quantum forms for the discrete $\mathrm{P}_{\mathrm{I}}, \mathrm{P}_{\mathrm{II}}, \mathrm{P}_{\mathrm{III}}$ and $\mathrm{P}_{\mathrm{IV}}$.

\section{Non-commuting variables: some basic relations}

Before proceeding to the derivation of quantum discrete Painlevé equations we should present a summary of our findings in [9]. Our derivation of quantum continuous Painlevé equations consists in extending the symmetrical form of Painlevé equations proposed by Noumi and Yamada [11] (see also Willox et al. [15]) to non-commuting objects. In the case of the quantum $\mathrm{P}_{\mathrm{II}}$ equation we introduce three unknown operators $f_{0}, f_{1}, f_{2}$ of $t$ and two parameters $\alpha_{0}, \alpha_{1}$ in the complex number field $\mathbb{C}$. The commutation rules are

$$
\left[f_{1}, f_{0}\right]=2 \hbar f_{2}, \quad\left[f_{0}, f_{2}\right]=\left[f_{2}, f_{1}\right]=\hbar .
$$

(In a more physical parlance we can say that the $\hbar$ appearing in the commutation relations is the Planck constant).

Generalising these relations to more objects so as to describe higher quantum Painlevé equations is straightforward. For a positive number $l$ greater than 1 , we introduce $l+1$ c-number parameters $\alpha_{i}(0 \leq i \leq l)$ and unknown operators $f_{i}(0 \leq i \leq l)$ with commutation relations

$$
\left[f_{i}, f_{i+1}\right]=\hbar, \quad\left[f_{i}, f_{j}\right]=0 \quad \text { otherwise }
$$

where the indices $0,1, \ldots, l$ are understood as elements of $\mathbb{Z} /(l+1) \mathbb{Z}$.

In the case of the quantum $\mathrm{P}_{\mathrm{II}}$ equation, the evolution equations with respect to $t$ for the unknown operators $f_{i}$ are

$$
\partial_{t} f_{0}=f_{0} f_{2}+f_{2} f_{0}+\alpha_{0}, \quad \partial_{t} f_{1}=-f_{1} f_{2}-f_{2} f_{1}+\alpha_{1}, \quad \partial_{t} f_{2}=f_{1}-f_{0},
$$

and in the case of the quantum $\mathrm{P}_{\mathrm{IV}}$ equation, the quantum $\mathrm{P}_{\mathrm{V}}$ equation and higher Painlevé equations, the evolution equations with respect to $t$ for the unknown operators $f_{i}$ are: for $l=2 n$ 
$(n \geq 1)$,

$$
\partial_{t} f_{i}=f_{i}\left(\sum_{1 \leq r \leq n} f_{i+2 r-1}\right)-\left(\sum_{1 \leq r \leq n} f_{i+2 r}\right) f_{i}+\alpha_{i},
$$

and for $l=2 n+1(n \geq 1)$,

$$
\begin{aligned}
\partial_{t} f_{i}= & f_{i}\left(\sum_{1 \leq r \leq s \leq n} f_{i+2 r-1} f_{i+2 s}\right)-\left(\sum_{1 \leq r \leq s \leq n} f_{i+2 r} f_{i+2 s+1}\right) f_{i} \\
& +\left(\frac{k}{2}-\sum_{1 \leq r \leq n} \alpha_{i+2 r}\right) f_{i}+\alpha_{i} \sum_{1 \leq r \leq n} f_{i+2 r},
\end{aligned}
$$

where $k=\alpha_{0}+\cdots+\alpha_{l}$. These (non-commutative) differential systems are quantization of nonlinear ordinary differential systems proposed by Noumi and Yamada in [11], and equal to quantization of $\mathrm{P}_{\mathrm{IV}}$ and $\mathrm{P}_{\mathrm{V}}$ for $l=2$ and $l=3$, respectively.

In the introduction we have stressed the necessity for the commutation rule to be consistent with the evolution. All systems here are consistent with the corresponding evolution $\partial_{t}$, namely the evolution $\partial_{t}$ preserves the commutation relations (2) for the case of the quantum $\mathrm{P}_{\mathrm{II}}$ equation and (3) for the other quantum Painlevé equations.

The non-commutative differential systems (4), (5) and (6) admit the affine Weyl group actions of type $A_{l}^{(1)}$, respectively, as well as the classical case. The actions are as follows. In the case of the quantum $\mathrm{P}_{\mathrm{II}}$ equation, we have

$$
\begin{aligned}
& s_{0}\left(f_{0}\right)=f_{0}, \quad s_{0}\left(f_{1}\right)=f_{1}-f_{2} \frac{\alpha_{0}}{f_{0}}-\frac{\alpha_{0}}{f_{0}} f_{2}-\frac{\alpha_{0}^{2}}{f_{0}^{2}}, \quad s_{0}\left(f_{2}\right)=f_{2}+\frac{\alpha_{0}}{f_{0}}, \\
& s_{1}\left(f_{0}\right)=f_{0}+f_{2} \frac{\alpha_{1}}{f_{1}}+\frac{\alpha_{1}}{f_{1}} f_{2}-\frac{\alpha_{1}^{2}}{f_{1}^{2}}, \quad s_{1}\left(f_{1}\right)=f_{1}, \quad s_{1}\left(f_{2}\right)=f_{2}-\frac{\alpha_{1}}{f_{1}}, \\
& s_{0}\left(\alpha_{0}\right)=-\alpha_{0}, \quad s_{0}\left(\alpha_{1}\right)=\alpha_{1}+2 \alpha_{0}, \quad s_{1}\left(\alpha_{0}\right)=\alpha_{0}+2 \alpha_{1}, \quad s_{1}\left(\alpha_{1}\right)=-\alpha_{1}, \\
& \pi\left(f_{0}\right)=f_{1}, \quad \pi\left(f_{1}\right)=f_{0}, \quad \pi\left(f_{2}\right)=-f_{2}, \quad \pi\left(\alpha_{0}\right)=\alpha_{1}, \quad \pi\left(\alpha_{1}\right)=\alpha_{0} .
\end{aligned}
$$

The actions $s_{0}, s_{1}$ and $\pi$ preserve the commutation relations (2) and give a representation of the extended affine Weyl group of type $A_{1}^{(1)}$, namely, they satisfy the relations

$$
s_{i}^{2}=1, \quad \pi^{2}=1, \quad \pi s_{i}=s_{i+1} \pi .
$$

Moreover the actions of $s_{0}, s_{1}$ and $\pi$ commute with the differentiation $\partial_{t}$, that is, they are Bäcklund transformations of the quantum $\mathrm{P}_{\mathrm{II}}$ equation.

In the case of the quantum $\mathrm{P}_{\mathrm{IV}}$ equation $(l=2)$, the quantum $\mathrm{P}_{\mathrm{V}}$ equation $(l=3)$ and higher quantum Painlevé equations $(l \geq 4)$, for $i, j=0,1, \ldots, l(l \geq 2)$ we have

$$
s_{i}\left(f_{j}\right)=f_{j}+\frac{\alpha_{i}}{f_{i}} u_{i j}, \quad s_{i}\left(\alpha_{j}\right)=\alpha_{j}-\alpha_{i} a_{i j}, \quad \pi\left(f_{j}\right)=f_{j+1}, \quad \pi\left(\alpha_{j}\right)=\alpha_{j+1},
$$

where

$$
\begin{aligned}
& u_{i, i \pm 1}= \pm 1, \quad u_{l 0}=1, \quad u_{0 l}=-1, \quad u_{i j}=0 \quad \text { otherwise } \\
& a_{i i}=2, \quad a_{i, i \pm 1}=-1, \quad a_{l 0}=a_{0 l}=-1, \quad a_{i j}=0 \quad \text { otherwise. }
\end{aligned}
$$

The actions of $s_{i}(i=0,1, \ldots, l)$ and $\pi$ preserve the commutation relations (3) and give representations of the extended affine Weyl groups of type $A_{l}^{(1)}$, namely, they satisfy the relations

$$
s_{i}^{2}=1, \quad\left(s_{i} s_{i+1}\right)^{3}=1, \quad s_{i} s_{j}=s_{j} s_{i} \quad(j \neq i \pm 1), \quad \pi^{l+1}=1, \quad \pi s_{i}=s_{i+1} \pi .
$$


Moreover the actions of $s_{i}(i=0,1, \ldots, l)$ and $\pi$ commute with the differentiation $\partial_{t}$, that is, they are Bäcklund transformations of the corresponding non-commutative differential systems.

We stress that the auto-Bäcklund transformations preserve the commutation relations in each case so that a discrete evolution which is constructed from these auto-Bäcklund transformations also preserves the commutation relations.

We remark that each differential system (4), (5) or (6) has relations

$$
\partial_{t}\left(f_{0}+f_{1}+f_{2}^{2}\right)=k
$$

for the quantum $\mathrm{P}_{\mathrm{II}}$ case,

$$
\partial_{t}\left(\sum_{r=0}^{l} f_{r}\right)=k
$$

for the $l=2 n(n \geq 1)$ case and

$$
\partial_{t}\left(\sum_{r=0}^{l} f_{2 r}\right)=\frac{k}{2} \sum_{r=0}^{l} f_{2 r}, \quad \partial_{t}\left(\sum_{r=0}^{l} f_{2 r+1}\right)=\frac{k}{2} \sum_{r=0}^{l} f_{2 r+1}
$$

for the $l=2 n+1(n \geq 1)$ case, where $k=\alpha_{0}+\alpha_{1}+\cdots+\alpha_{l}$. For simplicity, we normalize $k=1$ in the following.

\section{The continuous quantum Painlevé II and the related discrete equation}

As explained in Section 2 the Painlevé II case can be obtained with three non-commuting objects $f_{0}, f_{1}, f_{2}$ as described in (2) and parameters $\alpha_{0}, \alpha_{1}$. From the dynamical equations

$$
\begin{aligned}
& f_{0}^{\prime}=f_{0} f_{2}+f_{2} f_{0}+\alpha_{0}, \\
& f_{1}^{\prime}=-f_{1} f_{2}-f_{2} f_{1}+\alpha_{1}, \\
& f_{2}^{\prime}=f_{1}-f_{0},
\end{aligned}
$$

(where the prime' denotes differentiation with respect to $t$ ), eliminating $f_{0}, f_{1}$ we obtain the equation for the quantum $\mathrm{P}_{\mathrm{II}}$

$$
f_{2}^{\prime \prime}=2 f_{2}^{3}-t f_{2}+\alpha_{1}-\alpha_{0}
$$

which has the same expression as the commutative $\mathrm{P}_{\mathrm{II}}$. On the other hand, if we eliminate $f_{0}, f_{2}$ we find the quantum version of $\mathrm{P}_{34}$. We obtain

$$
f_{1}^{\prime \prime}=\frac{1}{2} f_{1}^{\prime} f_{1}^{-1} f_{1}^{\prime}-4 f_{1}^{2}+2 t f_{1}-\frac{1}{2}\left(\alpha_{1}^{2}-\hbar^{2}\right) f_{1}^{-1}
$$

The difference of this "quantum" version with the commutative $\mathrm{P}_{34}$ is clearly seen in the term quadratic in the first derivative (which would have been $f_{1}^{\prime 2} / f_{1}$ in the commutative case) but also in the last term. As a matter of fact, the coefficient of the term proportional to $1 / f_{1}$ is a perfect square in the commutative case. The appearance of the $-\hbar^{2}$ in the $\alpha_{1}^{2}-\hbar^{2}$ coefficient is a consequence of the non-commutative character of the $f_{i}$ 's.

In order to derive the discrete equation obtained as a contiguity relation of the solutions of the quantum $\mathrm{P}_{\mathrm{II}}$ we shall use the relations (7) presented in the previous section. We define an 
evolution in the parameter space of $\mathrm{P}_{\mathrm{II}}$ by $\bar{f} \equiv s_{1} \pi f$ and as a consequence the reverse evolution is $\underline{f} \equiv \pi s_{1} f$. Using the relations (7) we find

$$
\bar{f}_{2}+f_{2}=\alpha_{1} f_{1}^{-1}
$$

and

$$
f_{1}+f_{1}=t-f_{2}^{2}
$$

In the same way we compute the effect of the transformations on the parameters $\alpha$. We find $\bar{\alpha}_{1}=\alpha_{1}+1$ (and similarly $\underline{\alpha}_{1}=\alpha_{1}-1$ ). Thus applying $n$ times the transformation $s_{1} \pi$ on $\alpha_{1}$, we find that $\alpha_{1}$ becomes $\alpha_{1}+n$. We eliminate $f_{1}$ between the two equations (9) and (10) and obtain an equation governing the evolution in the parameter space. We find

$$
\alpha_{1}\left(\bar{f}_{2}+f_{2}\right)^{-1}+\alpha_{-}\left(f_{2}+f_{2}\right)^{-1}=t-f_{2}^{2} .
$$

This is the quantum version of the equation known, in the commutative case, as the alternate discrete Painlevé I.

\section{The continuous quantum Painlevé IV and the related discrete equation}

We turn now to the case of the quantum $\mathrm{P}_{\mathrm{IV}}$ which is obtained from the equations presented in Section 2 for $l=2$. Again we have three dependent variables $f_{0}, f_{1}, f_{2}$. The quantum continuous $\mathrm{P}_{\mathrm{IV}}$ equation is

$$
\begin{aligned}
& f_{0}^{\prime}=f_{0} f_{1}-f_{2} f_{0}+\alpha_{0}, \\
& f_{1}^{\prime}=f_{1} f_{2}-f_{0} f_{1}+\alpha_{1}, \\
& f_{2}^{\prime}=f_{2} f_{0}-f_{1} f_{2}+\alpha_{2},
\end{aligned}
$$

which is very similar to the one obtained in the commutative case. It is interesting to eliminate $f_{0}$ and $f_{2}$ and give the equation for $f_{1}$. After a somewhat lengthy calculation we find

$$
f_{1}^{\prime \prime}=\frac{1}{2} f_{1}^{\prime} f_{1}^{-1} f_{1}^{\prime}+\frac{3}{2} f_{1}^{3}-2 t f_{1}^{2}+\left(\frac{t^{2}}{2}+\alpha_{2}-\alpha_{0}\right) f_{1}-\frac{1}{2}\left(\alpha_{1}^{2}-\hbar^{2}\right) f_{1}^{-1}
$$

where we have used the identity $\sum_{i} f_{i}=t$. Again we remark that this equation, with respect to the commutative $\mathrm{P}_{\mathrm{IV}}$, contains an explicit quantum correction.

Before proceeding to the construction of the discrete system related to this equation we derive some auxiliary results. Starting from the action of $s_{i}$ on $f_{j}$, which from (8) is just $s_{i}\left(f_{j}\right)=f_{j}+\alpha_{i} f_{i}^{-1} u_{i j}$ we find $s_{i}\left(\sum_{j} f_{j}\right)=\sum_{j} f_{j}$ because $\sum_{j} u_{i j}=0$. Similarly $\pi\left(\sum_{j} f_{j}\right)=$ $\sum_{j} f_{j}$. Thus $\sum_{j} f_{j}$ is conserved under any combination of the transformations $\pi$ and $s_{i}$ of (8).

We can now define the evolution in the parameter space just as we did in the case of the quantum $\mathrm{P}_{\mathrm{II}}$. We put $\bar{f} \equiv s_{1} s_{0} \pi f$ and for the reverse evolution is $f \equiv \pi^{-1} s_{0} s_{1} f$. Using the relations (8) we find

$$
\bar{f}_{2}+f_{1}+f_{2}=t-\frac{\alpha_{1}}{f_{1}}
$$

and similarly

$$
f_{1}+f_{2}+f_{-}=t+\frac{\alpha_{2}}{f_{2}} .
$$


We now study the effect of the up-shift operator on $\alpha_{1}$ and $-\alpha_{2}$ which play the role of the independent variable. (The minus sign in front of $\alpha_{2}$ guarantees that the two equations have the same form). Using the relations in (8) and the fact that the sum of the $\alpha$ 's is constant we find $\bar{\alpha}_{1}=\alpha_{1}+1$ and $\left(-\bar{\alpha}_{2}\right)=\left(-\alpha_{2}\right)+1$. Thus incrementing the independent variable under repeated applications of the up-shift operator leads to a linear dependence on the number of iterations. Still, since the starting point is different we have two free parameters. Thus the system (11), (12) is exactly the quantum analogue of the equation known (in the commutative case) as the "asymmetric discrete Painlevé I", which is, in fact, a discrete form of $\mathrm{P}_{\mathrm{II}}$ [5].

\section{The continuous quantum Painlevé V and the related discrete systems}

Finally we examine the case of the quantum $\mathrm{P}_{\mathrm{V}}$, corresponding to the case $l=3$ in Section 2 . The quantum continuous $\mathrm{P}_{\mathrm{V}}$ equation is

$$
\begin{aligned}
& f_{0}^{\prime}=f_{0} f_{1} f_{2}-f_{2} f_{3} f_{0}+\left(\frac{1}{2}-\alpha_{2}\right) f_{0}+\alpha_{0} f_{2}, \\
& f_{1}^{\prime}=f_{1} f_{2} f_{3}-f_{3} f_{0} f_{1}+\left(\frac{1}{2}-\alpha_{3}\right) f_{1}+\alpha_{1} f_{3}, \\
& f_{2}^{\prime}=f_{2} f_{3} f_{0}-f_{0} f_{1} f_{2}+\left(\frac{1}{2}-\alpha_{0}\right) f_{2}+\alpha_{2} f_{0}, \\
& f_{3}^{\prime}=f_{3} f_{0} f_{1}-f_{1} f_{2} f_{3}+\left(\frac{1}{2}-\alpha_{1}\right) f_{3}+\alpha_{3} f_{1} .
\end{aligned}
$$

We remark that $f_{0}^{\prime}+f_{2}^{\prime}=\left(f_{0}+f_{2}\right) / 2$ and similarly $f_{1}^{\prime}+f_{3}^{\prime}=\left(f_{1}+f_{3}\right) / 2$. Thus two of the variables can be easily eliminated by introducing explicitly the time variable through the exponential $e^{t / 2}$. Just as in the case of $\mathrm{P}_{\mathrm{III}}$ and $\mathrm{P}_{\mathrm{IV}}$ we can eliminate one further variable and obtain the quantum form of $\mathrm{P}_{\mathrm{V}}$ expressed in terms of a single variable. In the present case it is more convenient to introduce an auxiliary variable $w=1-e^{t / 2} / f_{0}$. We obtain thus for $w$ the equation

$$
\begin{aligned}
w^{\prime \prime}= & w^{\prime}\left(\frac{1}{w-1}+\frac{1}{2 w}\right) w^{\prime}+(w-1)^{2}\left(\frac{\alpha_{0}^{2}-\hbar^{2}}{2} w+\frac{\hbar^{2}-\alpha_{2}^{2}}{2 w}\right) \\
& +\left(\alpha_{3}-\alpha_{1}\right) e^{t} w+e^{2 t} \frac{w(w+1)}{2(1-w)} .
\end{aligned}
$$

As in the previous cases, the equation (13) contains an explicit quantum correction, with respect to the commutative $\mathrm{P}_{\mathrm{V}}$, as well as a symmetrisation of the term quadratic in the first derivative.

Just as in the case of commutative $\mathrm{P}_{\mathrm{V}}$ we can define several different evolutions in the parameter space giving rise to different discrete equations. For the first equation we introduce the first up-shift operator $R=\pi s_{3} s_{2} s_{1}$ from (8) for $l=3$. If we define $x=f_{0}+f_{2}$ and $y=f_{1}+f_{3}$ a careful application of the rules (8) shows that $R x=y$ and $R y=x$. Next we introduce an auxiliary variable $g$ defined by $g=f_{3}-\alpha_{0} f_{0}^{-1}=y-f_{1}-\alpha_{0} f_{0}^{-1}$ and we seek an equation in terms of the variables $f_{1}, f_{0}, g$. Calling $\bar{f} \equiv R f$, we find

$$
\bar{f}_{1}+f_{0}=x-\frac{\alpha_{0}+\alpha_{3}}{g}
$$

complemented by the equation coming from the definition of $g$

$$
f_{1}+g=y-\frac{\alpha_{0}}{f_{0}}
$$


and finally

$$
g+f_{0}=x+\frac{\alpha_{1}}{f_{1}}
$$

In order to introduce the proper independent variable we consider the action of $R$ on the $\alpha$ 's: $\bar{\alpha}_{0}=\alpha_{0}+1, \bar{\alpha}_{1}=\alpha_{1}-1, \bar{\alpha}_{2}=\alpha_{2}$ and $\bar{\alpha}_{3}=\alpha_{3}$. Thus we can choose $z=\alpha_{0}$ (which grows linearly with the successive applications of $R$ ). The system (14), (15) and (16) is the quantum analogue of the asymmetric, ternary, discrete Painlevé I, which, as we have shown in [3] is a discrete form of the Painlevé IV equation. (At this point we should point out that the alternating constants $x, y$ do not introduce an extra degree of freedom. As a matter of fact by choosing an appropriate gauge of the dependent variables and rescaling of the independent ones we can bring these constants to any non-zero value).

For the second equation we introduce a new up-shift operator $T=s_{1} \pi s_{3} s_{2}$, the action of which is again obtained with the help of (8). The variables $x$ and $y$ are defined in the same way as in the previous paragraph and again we have $T x=y$ and $T y=x$. An auxiliary variable is necessary in this case also and thus we introduce $h=f_{3}+\alpha_{2} f_{2}^{-1}$. We denote the action of $T$ by a tilde: $\tilde{f} \equiv T f$. We seek an equation for $f_{1}, f_{2}, h$. We find

$$
\underset{\sim}{f_{1}}+f_{2}=x+\frac{\alpha_{2}+\alpha_{3}}{h} .
$$

The definition of $h$ implies

$$
h+f_{1}=y+\frac{\alpha_{2}}{f_{2}}
$$

and finally we have

$$
f_{2}+\tilde{h}=x-\frac{\alpha_{1}}{f_{1}} .
$$

The action of $T$ on the $\alpha$ 's is $\tilde{\alpha}_{0}=\alpha_{0}, \tilde{\alpha}_{1}=\alpha_{1}+1, \tilde{\alpha}_{2}=\alpha_{2}-1$ and $\tilde{\alpha}_{3}=\alpha_{3}$.

Combining the two systems above we can obtain a nicer, and more familiar, form. First, comparing (16) and (19), we find that $\underline{g}=f_{2}+\alpha_{1} f_{1}^{-1}=x-\tilde{h}=T(y-h)$, or equivalently $g=R T(y-h)$. We are thus led to introduce the operator $S=R T$, and we denote its action by a "hat" accent $\hat{f}=S f$. Subtracting (15) from (18) and eliminating $g$ through the relation $g=y-\hat{h}$, we obtain

$$
h+\hat{h}=y+\frac{\alpha_{0}}{f_{0}}-\frac{\alpha_{2}}{f_{0}-x} .
$$

For the second equation we start from (17) and apply the operator $R T$ to it. We find

$$
\bar{f}_{1}+\hat{f}_{2}=x-\frac{\alpha_{0}+\alpha_{1}}{\hat{h}}
$$

where we have used the fact that $R T\left(\alpha_{2}+\alpha_{3}\right)=-\left(\alpha_{0}+\alpha_{1}\right)$. Next we subtract (21) from (14), use the definition of $x$ in order to eliminate $f_{2}$ and find finally

$$
f_{0}+\hat{f}_{0}=x+\frac{\alpha_{0}+\alpha_{3}}{\hat{h}-y}+\frac{\alpha_{0}+\alpha_{1}}{\hat{h}} .
$$

A careful application of the rules (8) shows that the action of the operator $R T$ on each of the numerators of the fractions in the right hand side of (20) and (22) results in an increase by exactly 1 and thus an independent variable linear in the number of iterations of $R T$ can be introduced. As in the case of the first system (14), (15) and (16) presented in this section 
the alternating constants $x, y$ do not introduce an extra degree of freedom. A better choice of the dependent variables would be $h \rightarrow h-y / 2$ and $f_{0} \rightarrow f_{0}-x / 2$. Moreover, by choosing an appropriate gauge of the dependent variables and rescaling of the independent ones we can bring these constants to any non-zero value, for instance $x=y=2$. The system now becomes

$$
\begin{aligned}
& h+\hat{h}=\frac{\alpha_{0}}{f_{0}+1}-\frac{\alpha_{2}}{f_{0}-1}, \\
& f_{0}+\hat{f}_{0}=\frac{\alpha_{0}+\alpha_{3}}{\hat{h}-1}+\frac{\alpha_{0}+\alpha_{1}}{\hat{h}+1} .
\end{aligned}
$$

Under this form one recognizes immediately the structure of the "asymmetric discrete Painlevé II" equation, introduced in [2] and which is a discrete analogue of $\mathrm{P}_{\mathrm{III}}$. Thus (23) and (24) constitute the quantum extension of the latter.

\section{Conclusions}

In this paper, we have analysed the "quantum" forms of Painlevé equations derived by one of us (HN) in [9]. The derivation consists in extending the symmetrical form of Painlevé equations proposed by Noumi and Yamada to non-commuting variables. We have focused here on $\mathrm{P}_{\mathrm{II}}, \mathrm{P}_{\mathrm{IV}}$ and $\mathrm{P}_{\mathrm{V}}$ and derived their more "familiar" forms expressed in terms of a single variable. The noncommutative character manifests itself in the fact that the dependent function does not commute with its first derivative. As a consequence (and despite the fact that a symmetrised form of the term quadratic in the first derivative is used) explicit quantum corrective terms appear in the equation, proportional to the square of the Planck constant. Using the auto-Bäcklund transformations of the continuous Painlevé equations we derive their contiguity relations which are just the quantum forms for the discrete $\mathrm{P}_{\mathrm{I}}, \mathrm{P}_{\mathrm{II}}, \mathrm{P}_{\mathrm{III}}$ and $\mathrm{P}_{\mathrm{IV}}$.

\section{References}

[1] Fokas A., Grammaticos B., Ramani A., From continuous to discrete Painlevé equations, J. Math. Anal. Appl. 180 (1993), 342-360.

[2] Grammaticos B., Nijhoff F.W., Papageorgiou V., Ramani A., Satsuma J., Linearization and solutions of the discrete Painlevé III equation, Phys. Lett. A 185 (1994), 446-452, solv-int/9310003.

[3] Grammaticos B., Ramani A., Papageorgiou V., Discrete dressing transformations and Painlevé equations, Phys. Lett. A 235 (1997), 475-479.

[4] Grammaticos B., Ramani A., Papageorgiou V., Nijhoff F., Quantization and integrability of discrete systems, J. Phys. A: Math. Gen. 25 (1992), 6419-6427.

[5] Grammaticos B., Ramani A., From continuous Painlevé IV to the asymmetric discrete Painlevé I, J. Phys. A: Math. Gen. 31 (1998), 5787-5798.

[6] Hietarinta J., Classical versus quantum integrability, J. Math. Phys. 25 (1984), 1833-1840.

[7] Hietarinta J., Grammaticos B., On the $\hbar^{2}$-correction terms in quantum integrability, J. Phys. A: Math. Gen. 22 (1989), 1315-1322.

[8] Jimbo M., Miwa T., Monodromy preserving deformation of linear ordinary differential equations with rational coefficients. II, Phys. D 2 (1981), 407-448.

[9] Nagoya H., Quantum Painlevé systems of type $A_{l}^{(1)}$, Internat. J. Math. 15 (2004), 1007-1031, math.QA/0402281.

[10] Nagoya H., Quantum Painlevé systems of type $A_{n-1}^{(1)}$ with higher degree Lax operators, Internat. J. Math. 18 (2007), 839-868.

[11] Noumi M., Yamada Y., Higher order Painlevé equations of type $A_{l}^{(1)}$, Funkcial. Ekvac. 41 (1998), 483-503, math.QA/9808003. 
[12] Novikov S.P., Quantization of finite-gap potentials and a nonlinear quasiclassical approximation that arises in nonperturbative string theory, Funct. Anal. Appl. 24 (1990), 296-306.

[13] Quispel G.R.W., Nijhoff F.W., Integrable two-dimensional quantum mappings, Phys. Lett. A 161 (1992), 419-422.

[14] Quispel G.R.W., Roberts J.A.G., Thompson C.J., Integrable mappings and soliton equations. II, Phys. D 34 (1989), 183-192.

[15] Ramani A., Willox R., Grammaticos B., Carstea A.S., Satsuma J., Limits and degeneracies of discrete Painlevé equations: a sequel, Phys. A 347 (2005), 1-16.

[16] Ramani A., Tamizhmani T., Grammaticos B., Tamizhmani K.M., The extension of integrable mappings to non-commuting variables, J. Nonlinear Math. Phys. 10 (2003), suppl. 2, 149-165. 